\title{
Physical Activity Sensing via Stand-Alone WiFi Device
}

\author{
Wenda Li*, Robert. J. Piechocki ${ }^{\dagger}$, Karl Woodbridge ${ }^{\ddagger}$, Kevin Chetty* \\ ${ }^{*}$ Department of Security and Crime Science, University College London, UK \\ ${ }^{\dagger}$ Department of Electrical and Electronic Engineering, University of Bristol, UK \\ $\ddagger$ Department of Electronic and Electrical Engineering, University College London, UK \\ \{wenda.li, k.chetty, k.woodbridge\}@ucl.ac.uk, r.j.piechocki@bristol.ac.uk
}

\begin{abstract}
WiFi signals for physical activity sensing shows promising potential for many healthcare applications due to its potential for recognising various everyday activities, non-invasive nature and low intrusion on privacy. Traditionally, WiFi-based sensing uses the Channel State Information (CSI) from an offthe-shelf WiFi Access Point (AP) which transmits signals that have high pulse repetition frequencies. However, when there are no users on the communication network only beacon signals are transmitted from the WiFi AP which significantly deteriorates the sensitivity and specificity of such systems. Surprisingly WiFi based sensing under these conditions have received little attention given that WiFi APs are frequently in idle state. This paper presents a practical system based on passive radar technique which does not require any special setup or preset firmware and able to work with any commercial WiFi device. To cope with the low density of beacon signal, a modified Cross Ambiguity Function (CAF) has been proposed to reduce redundant samples in the recorded. In addition, an external device has been developed to send WiFi probe request signals and stimulate an idle AP to transmit WiFi probe responses thus generate usable transmission signals for sensing applications without the need to authenticate and join the network. Experimental results prove that proposed concept can significantly improve activity detection and is an ideal candidate for future healthcare and security applications.
\end{abstract}

Index Terms-WiFi Sensing, Physical Activity, Modified Cross Ambiguity Function, Stand-Alone WiFi Device

\section{INTRODUCTION}

Over the last decade, there have been increasing concerns about the impacts which the ageing population will have on long-term conditions such as diabetes, mental health, obesity and cardiovascular disease because of their importance in social care and security [1], and subsequent demands in financial and human resource constraints on healthcare services. Daily activities and behaviors sensing in a residential area are important for both long-term and short-time tasks such as life patterns and falls. Such measurements are extremely helpful for preventing health risks and chronic diseases, in which early interventions are critical. Besides traditional approaches that rely on wearable sensors or cameras, WiFi-based sensing technology is considered as an ideal solution for multiple indoor monitoring purposes. The reason is, in part, due to the passive nature of the $\mathrm{WiFi}$ sensing and pervasive nature of WiFi AP in both residential and work settings. Additionally, wireless signals cannot be used to generate images the people they are monitoring which alleviates many concerns and barriers around privacy issues.

The fundamental principle of $\mathrm{WiFi}$ sensing is that the movement of a person will affect the communication channel in terms of signal attenuation, frequency shift and propagation paths. As a result, the communication channel is time-varying with the physical activities that can be used for monitoring purposes. For example, the authors in [2] propose a Received Signal Strength (RSS) based method for localization, whilst the work in [3] extends this method for activity recognition. However RSS-based systems require intensive offline training to assess the surrounding environment and also suffer from coarse resolution. As a result of this, researchers focused on obtaining more accurate and detailed information by leveraging the CSI from commercially available network cards [4]. In [5], phase information together with RSS profiles have been used to identify people falling in healthcare applications, while a subspace-based method in [6] collects the Angle of Arrival (AoA) and Time of Arrival (ToA) information for fine-grained indoor localization. However, the above systems are all based on a full bandwidth WiFi signal is being transmitted which is not realistic for many scenarios, for example at night or in households that infrequently use the internet. Evaluation of beacon-only signal transmissions which occur in these situations when a WiFi AP is in an idle state has received little attention from researchers. Since the primary purpose of WiFi network is to connect devices, but the additional transmission of WiFi signal aims for sensing purpose will no doubt waste a considerable bandwidth and cause interference to the communication channel.

The work in [7] points that the default beacon interval in commodity WiFi AP is 100 Time Unit $(1 \mathrm{TU}=1024 \mathrm{~ns})$, which is not suitable for sensing. Cross Ambiguity Function (CAF) processing is a cross-correlation based technique between a surveillance and reference receiver channel that is employed in passive radar to generate Doppler information about targets of interest within the sensing area. However, it cannot properly function with the ultra-low-dense of beacon signal as it will be corrupted by the noise. Thus, a modified CAF mapping approach has been proposed to maximize the use of beacon signal and filter out the undesired noise. For this reason, an external-active device has been used to generate 

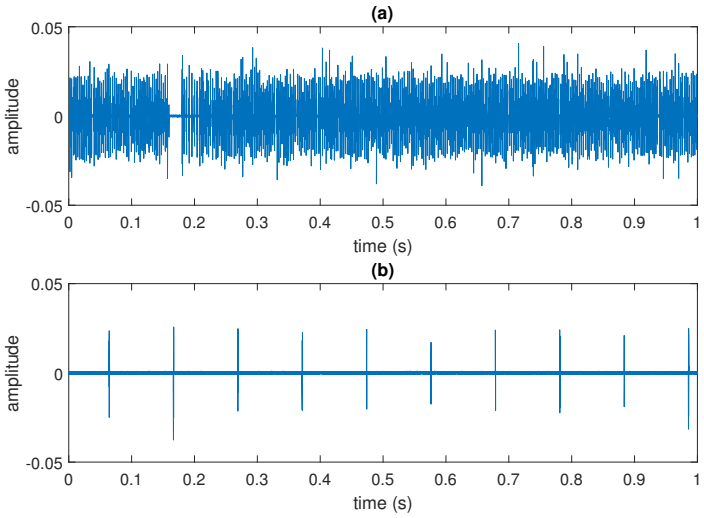

Fig. 1: An example of WiFi status (a) transmission status (full bandwidth WiFi signal) and (b) idle status (only beacon signal)

transmissions with significantly higher duty-cycles to facilitate more precise Doppler resolution. This device uses the probe request-response protocol in the $\mathrm{WiFi}$ standard which exchanges information between the WiFi AP and client devices. In addition, the probe protocol has allowances for repeat requests that can be used to stimulate any WiFi AP to transmit without authenticating through the network. Our processing also includes implementation of a modified CLEAN algorithm [8] which is used to remove the zero Doppler peak caused by the strong Direct Signal Interference (DSI) and a Constant False Alarm Rate (CFAR) for activity identification.

The rest of this paper is organized as follow: Section II outlines the operating model of WiFi AP; Section III presents the signal processing of the proposed system; Section IV describes the implementation of the device to activate $\mathrm{WiFi}$ AP remotely; Section V presents the experimental results acquired in a real-world environment. Finally we make some conclusions in Section VI.

\section{WiFi Signal Characteristics AND System FRAMEWORK}

\section{A. WiFi Status}

In passive sensing, the $\mathrm{WiFi}$ signal is considered as an independent pseudo-noise waveform and can be divided into transmission and idle status. As explained above, all current WiFi based sensing systems rely on the high data-rate $\mathrm{WiFi}$ signals to extract the CSI/Doppler information. Here we provide two examples of WiFi signal; the first is when the AP is transmitting data using OFDM modulations, and the second is from the beacon signal (default periodicity of $100 \mathrm{TU}$ ) when a WiFi AP is in its idle state. The data for both examples were captured for one second, and their time domain representations are illustrated in in Fig 1. As shown in the figure, the $\mathrm{WiFi}$ signal occupies almost full bandwidth (>90\%) during the transmission status with small time gap. This provides a large amount of effective data to be used by the passive radar system. In comparison, during idle status, the beacon signal has a small duty cycle with large spaces of redundancy. In fact,

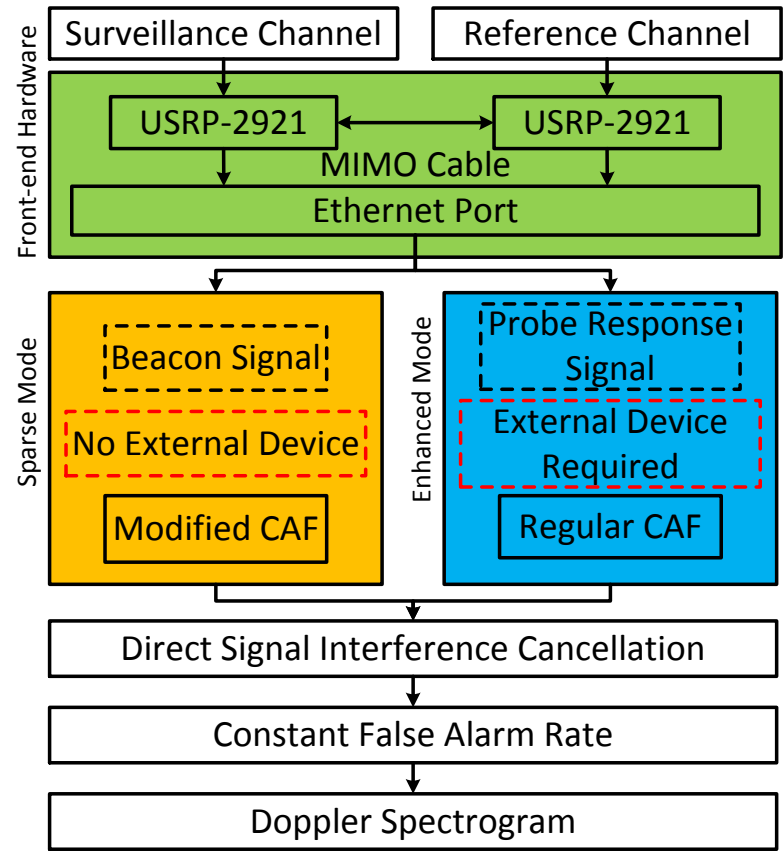

Fig. 2: Overall block diagram of the proposed system

manual measurements show that the duty cycle of the beacon signal is $0.42 \%$ of that of a data transmission signal. As a result, the effective data from the beacon signal is very limited, and therefore the classical CAF process will not function at this level. In this work, we focus on the solution for WiFi sensing during the idle status of a WiFi AP.

\section{B. Beacon and Probe Response Signal}

Both the beacon and probe response signal are management, not encrypted frames in IEEE 802.11 standard. The beacon signal is transmitted periodically to declare the presence of a WLAN with information about the network like modulation, code rate, compatible standard. Probe response signal is requested by a client device to provide information about the AP with similar information as beacon signal. On the other hand, the beacon signal is broadcast constantly from a WiFi $\mathrm{AP}$, whereas probe response signal is a response to the client device. Both signals can be used for opportunity sensing, but the quality depends on the amount of effective data.

\section{System Overview}

The overall block diagram of the system is presented in Fig 2. The surveillance and reference channels are measured using two Yagi antennas directed towards the surveillance area and WiFi AP respectively. The front-end hardware was built based on two NI USRP-2921 [9] for wireless signal acquisition and transferred to a computing unit (in this work, a laptop running LabVIEW) through a Gigabyte Ethernet. The proposed system has two modes termed Sparse mode and Enhanced mode. The sparse mode uses the original beacon signal and processed with modified CAF process for limited activity detection. The enhanced mode uses the probe response 
signal which is requested an external device for full activity detection. And since there is no sparse signal problem, the regular CAF is used in enhanced mode. Afterward, in postprocessing, a CLEAN algorithm and CFAR approach are used to remove the direct signal interference (DSI) and activity identification respectively.

\section{Signal Processing For Passive WiFi RADAR}

In IEEE 802.11 standard, signals are modulated by OFDM scheme [10]. This also applies to the beacon and probe response signal. Let the transmitted OFDM signal defined as:

$$
x(t)=\frac{1}{\sqrt{N}} \sum_{n=0}^{N-1} a_{n} e^{j 2 \pi n t}
$$

where $N$ is the number of OFDM symbols for each carries $a_{n}$ and $n$ is the index of OFDM symbol. The received signal $y(t)$ consists of both direct signal and target reflections. These reflections from a stationary cluster or a moving person can be described by a summation of delayed and phase shifted transmitted signal. The received signal can be written as:

$$
y(t)=\sum_{p} A_{p} e^{j 2 \pi f_{d} f_{c} t} x(t-\tau)+n(t)
$$

where $p$ is the number of reflected paths, and $A_{p}, \tau, f_{d}$ are the attenuation factor, delay, Doppler shift for $p$-th path respectively, $\mathrm{n}(\mathrm{t})$ is the Additive White Gaussian Noise (AWGN).

\section{A. Modified Cross Ambiguity Function}

CAF is a traditional processing in passive radar field to calculate time delay $\tau$ and Doppler shift $f_{d}$. The regular CAF equation can be written as:

$$
C A F\left(\tau, f_{d}\right)=\int_{T_{i}}^{0} x(t) y^{*}(t-\tau) e^{j 2 \pi f_{d} f_{c} t} d t
$$

where $T_{i}$ represents the integration time which defines the Doppler resolution as: $\Delta f=1 / T_{i}$. In the real implementation, a batch processing method [11] has been used for complexity reduction. This is achieved by segmenting a long sequence into several short batches so that the cross-correlation and FFT process are faster. However, Eq 3 cannot generate meaningful Doppler signature in the idle status (Fig 1(b)) due to the ultra-low-dense of beacon signal and noise. For this end, the modified $\mathrm{CAF}$ is proposed to maximize the usage on beacon signal. The idea is to synchronize and extract the beacon signal before them passing to the CAF. This can ensure only effective data are collected and processed. The beacon synchronization is implemented based on the energy curve $X_{i}$ which is calculated as the cumulative sum of amplitude values as defined in [12]. The separation of the beacon signal from the noise part is calculated as:

$$
X_{i}^{\prime}=X_{i}-i \delta=\sum_{k=0}^{i}\left(x_{k}^{2}-i \delta\right)
$$

where $i$ is the index of signal. $\delta$ is a negative trend and depends on the total energy of the selected signal $X_{N}$ with window length of $N$ (in this work, $N$ is the size of beacon interval) as determined by $\delta=\frac{X_{N}}{\alpha \cdot N}$. Suggest by [12], $\alpha=1$ has been selected for simplicity.

Afterward, the modified CAF with synchronized beacon signal can be written as:

$$
C A F\left(\tau, f_{d}\right)=\sum_{n=0}^{N_{b}-1} \int_{0}^{T_{b}} x_{n}(t) y_{n}^{*}(t-\tau) e^{j 2 \pi f_{d} f_{c} t} d t
$$

where, $N_{b}$ is the number of beacon, $T_{b}$ is the time duration of a beacon signal and $n$ is the index of beacon. To optimise performance, the beacon synchronization is designed based on the signal recorded in the reference channel as it has a higher SNR and is subject to less fluctuations that the signal measured in the surveillance channel. Note however, there is still interference in reference channel (for example, other packages, WiFi AP in the same frequency band), which may lead to synchronization distortions.

\section{B. Direct Signal Interference Cancellation}

One of the major interference for passive radar system is the strong direct signal from the transmitter. This causes an unwanted peak in the zero Doppler/range bin that may bury the target signal. Therefore, the CLEAN algorithm introduced in [13] has been used to remove DSI. Let $C A F^{k}\left(\hat{\tau}, \hat{f}_{d}\right)$ represents the cleaned CAF mapping at $k_{t h}$ iteration, it can be written as:

$C A F^{k}\left(\hat{\tau}, \hat{f}_{d}\right)=C A F^{k}\left(\tau, f_{d}\right)-\alpha^{k} C A F_{\text {self }}\left(\tau-T_{k}, f_{d}\right)$ where $\alpha^{k}$ is the maximum absolute value of $C A F^{k}\left(\tau, f_{d}\right)$, $T_{k}$ is the phase shift factor refers to the $\alpha^{k}$. CAF self is the self CAF over the reference channel. From observation, one iteration $(k=1)$ is sufficient for our system. The CLEAN algorithm is implemented the same way as CAF due to they share a similar structure.

\section{Constant False Alarm Rate}

After the DSI cancellation, we still observe considerable noise on the CAF. There are multiple possible reasons: incorrect beacon synchronization that output ineffective data, wrong CAF processing due to strong WiFi signal from other AP and other packet transmission interrupt the beacon synchronization. A simpler CFAR detector has been used to detect the target and filter out the noise.

$$
\Lambda=\frac{1}{N_{\tau} \cdot N_{f_{d}}} \sum_{i=1}^{R_{\tau}} \sum_{j=1}^{R_{f_{d}}} C A F\left(\tau_{i}, f_{d_{j}}\right)
$$

where $\Lambda$ is the threshold mapping for CAF. $i$ and $j$ are the index for range and Doppler bin, $N_{\tau}$ and $N_{f_{d}}$ are the training length in range and Doppler bin respectively. This threshold mapping is then used for normalizing the power and remove the noise as $P(i, j)=|C A F(i, j)|^{2} / \Lambda$. For $P(i, j)<1$ are considered as no motion and replaced with zeros, others are considered as activities.

The performance of both sparse and enhanced mode with the above signal processing will be given in Section V. 


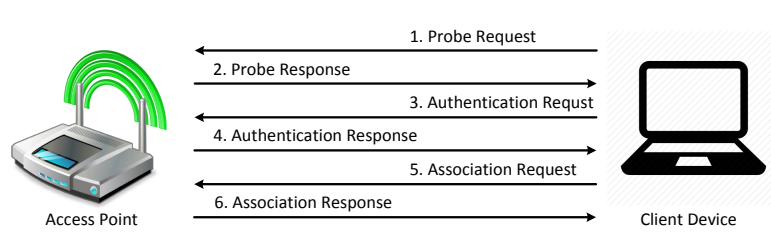

Fig. 3: Process of probe response signal

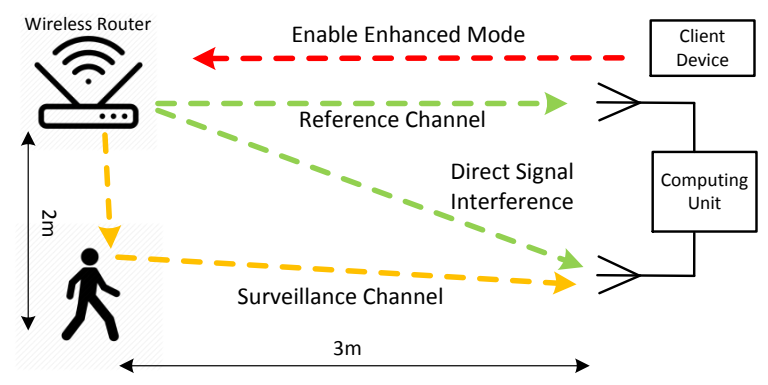

Fig. 4: Experiment Layout

\section{Enhanced Mode: Probe Response Signal}

Similar to the beacon signal, the probe response signal also has a bursty nature. However, they differ in that probe response is triggered by an external device which can be considered as an 'active' method, whereas beacon signal is broadcast 'passively'. Also, the frequency of probe response signal is controllable from the external device, and therefore it can be used to generate sufficient bandwidth for passive radar system without any type of AP configuration. In this system, probe response signal is only employed during the enhanced mode.

A Raspberry Pi running with Kali Linux is developed to stimulate a client device and continuously send probe requests frames to the target WiFi AP. It is processed according to the 802.11 probe protocol [14] as shown in Fig 3. In our tests, the maximum achievable frame rate for current setup is 75 probe requests per second (approximately $13 \mathrm{TU}$ ) which provides much higher density than the default setting of beacon signal (100 TU).

Despite, the enhanced mode can generate a large amount of effective data for our passive radar system. However, the bursty nature of probe response signal means there are still considerable time gaps when comparing to the WiFi transmission status (Fig 1(a)). These time gaps between the probe request/response could potentially result in insufficient correlation and generate noisy range-Doppler surface. It is expected that smaller time gap can further advance the WiFi sensing. Though, this can be achieved with alternative devices for a higher data rate.

\section{EXPERIMENTAL RESUltS}

In this section, experimental results are presented to show the sensing performance of the proposed system with a standalone WiFi AP. We used an EDIMAX AC 1200 router which transmits in $5 \mathrm{G}$ channel 44 , frequency of $5.22 \mathrm{GHz}$ and 20 $\mathrm{MHz}$ bandwidth. Note that, during the experiment, we do not
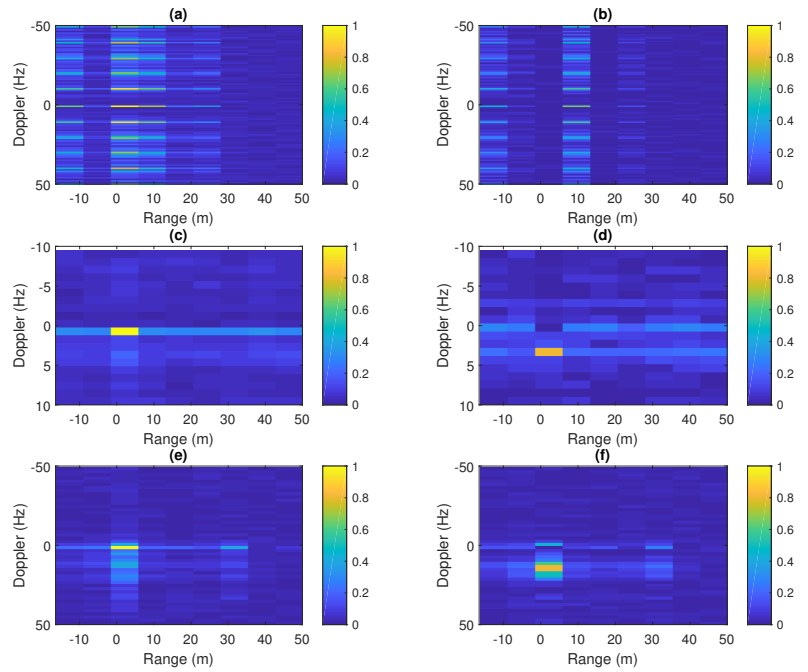

Fig. 5: An example of range-Doppler surface during WiFi idle status, (a) classical processing, (b) classical processing with interference removal, (c) sparse mode, (d) sparse mode with interference removal, (e) enhanced mode and (f) enhanced mode with interference removal

employ any type of bespoke configuration on the router, thus the beacon interval was fixed at default with the beacon dense as shown in Fig 1(b). The experiment layout is shown in Fig 4 in an office surrounding with furniture and equipment. One antenna was pointing to the router as the reference channel, and another antenna faced to the monitoring area as the surveillance channel for the CAF process. The external device was used during the enhancement mode to activated the probe response signal.

\section{A. Effectiveness of Proposed Signal processing}

During the experiments a person walked away from the surveillance antenna, and a single range-Doppler surface was obtained to demonstrate the effectiveness of proposed signal processing. This experiment was repeated under idle status and processed with classical passive radar processing (beacon signal with Eq 3), sparse mode (beacon signal with Eq 5), and enhanced mode (probe response signal with Eq 3). As it can be seen from Fig 5(a), the classical processing generates meshed range-Doppler surface and with a high level of noise, due to the sparse nature of beacon signal. There is still no meaningful Doppler information, after the noise removal process as shown in Fig 5(b).

For this reason, the sparse mode has been applied with the modified CAF. The result is plotted in Fig 5(c). The rangeDoppler surface is now cleared and a strong peak can be seen in the zero range/Doppler bin with a significant magnitude that buries other peaks. This is because of the sidelobes of antenna collects the direct signal and unwanted signal from other sources. The cleaned range-Doppler surface is shown in Fig 5(d), where the dominant peak has been successfully removed. As a result, the desired peak is present clearly on the map at 

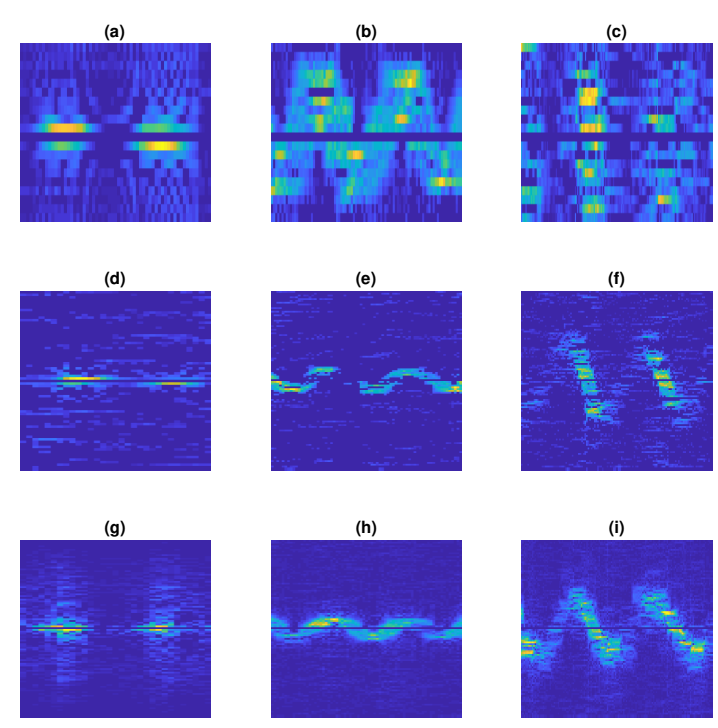

Fig. 6: Performance comparison: (a) sitting still (b) body swing and (c) walking in sparse mode; (d) sitting still (e) body swing and (f) walking in enhanced mode; (g) sitting still (h) body swing and (i) walking in transmission status

$10 \mathrm{~Hz}$ which corresponds to the person's movement. Note that, the boundary of Doppler is limited in sparse mode since there are only ten beacons available in each second which can be used in the batching process. This means the maximum detectable boundary is five Doppler bins for a second (in both positive and negative domain). To better demonstrate the Doppler pulse, the integration time $T_{I}$ for sparse mode was set as two seconds so that twenty beacons are available in the range-Doppler surface.

For the enhanced mode, it does not have the limitation on the Doppler boundary as the probe response signal are more frequently that does not need synchronization. In this mode, the number of Doppler bin was set at 100 for wider Doppler boundary. Consequently, it has higher resolution on Doppler pulse and with lower noise level when compared that to the sparse mode. Range-Doppler surfaces without\&with interference removal from enhanced mode are shown in both Fig 5(e) and (f). It shows the dominant peak in the zerorange and zero-Doppler bin has been suppressed with the CLEAN algorithm. Furthermore, both surfaces show more detailed Doppler information compare to the same process in sparse mode as (c) and (d).

\section{B. Performance Comparison}

Here, we compare the Doppler signature during the $\mathrm{WiFi}$ idle status with sparse and enhanced mode, and during the data transmission status. The Doppler signature is generated by picking columns containing the Doppler pulse from multiple range-Doppler surfaces. Three activity classes were measured for different level of velocity as sitting still at a distance of $50 \mathrm{~cm}$ (slow chest motion), body swing at a distance of 1 meter (medium part-body motion) and walking forward and backward from surveillance antenna within 3 meters at normal velocity (fast whole-body motion). During the transmission status, two client devices were set up to transfer data between each other in a high data-rate. Thus, the Doppler signature obtained in transmission status can be considered as the 'Gold Rule' due to the stable and continuously WiFi signal. It demonstrates the best result can be obtained at current settings which are used as the ground reference for other two modes in idle status.

The collected Doppler signatures are shown in Fig 6. As can be seen, sparse mode can give the correct Doppler pattern for breathing and body swing. The two peaks in (a) represent the Doppler change during the process of inhalation and exhalation of the chest, and the sinusoidal wave in (b) shows the periodical characteristics of body swings. Despite the fact that some noise remains in the spectrum, the overall Doppler shape is distinguishable. In comparison, Doppler signature for walking in (c) is difficult to interpret where its Doppler pulses span across the entire Doppler bin. This is due to the number of beacons induce a limitation on the maximum detectable velocity. Since the walking speed is faster than the body swing, therefore its Doppler goes beyond the Doppler boundary and appears in the other side of the Doppler domain. As a result, the sparse mode cannot correctly detect the activity with fast velocity. In such a case, WiFi idle status when processed in sparse mode can simply act as a motion detector.

A higher level of Doppler information can be obtained with the enhanced mode when additional information is required, for example activity recognition or contextual awareness [15]. With the higher density of probe response signal, the enhanced mode has a significant improvement on the Doppler signatures as shown in Fig 6(c), (d) and (e). It has the ability to detect all three activities correctly and shows similar performance to that in transmission status ( $\mathrm{f}$ ), (g) and (h) but with some discontinuity and low level of noise due to the time gap between probe response signal. This provides an attractive solution to collect quality Doppler information during the WiFi idle status. However, the use of enhanced mode means jammer on the communication channel. Thus the balance between the requirement on the $\mathrm{WiFi}$ sensing and the usage on bandwidth needs to be considered for a practical system.

\section{Demonstration of Long-Term Monitoring}

With the verification of Doppler signature from the previous experiment, we extend the analysis to long-term monitoring in order to demonstrate how a stand-alone WiFi AP can be used in application focused towards measuring patterns-oflife. This experiment was conducted in an office and tested under the idle status to avoid jammer in the communication channel. The surveillance antenna was pointing to a seat in the office with the experiment layout as shown in Fig 4. The measurement rate was set at 10 readings per second. The recorded Doppler spectrogram from 7AM until 7PM is plotted in Fig 7(a). As can be seen, there are several Doppler spikes in the spectrogram and most of them are concentrated between 10AM to 3PM. Fig 7(b) provides the number of 

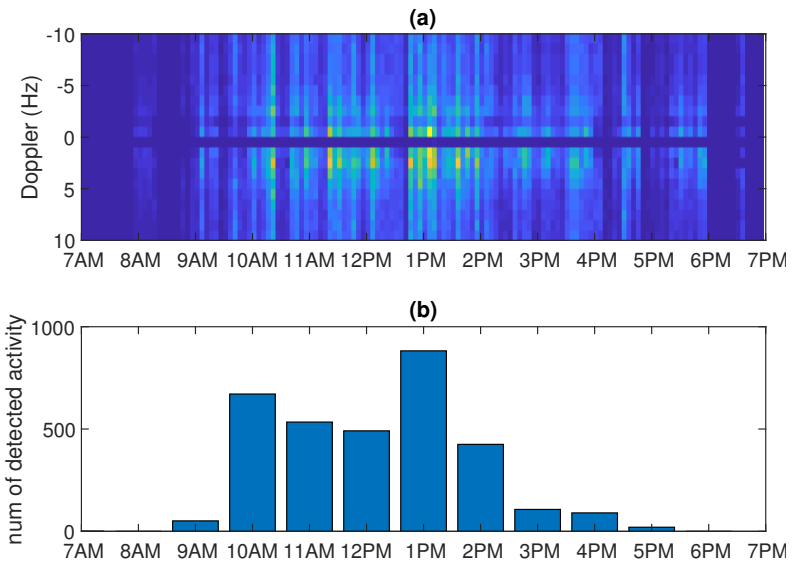

Fig. 7: An example of long period monitoring under sparse mode, (a) recorded Doppler spectrogram and (b) number of detected activity within each hour

TABLE I: Statistics of Activity Level

\begin{tabular}{|l|l|l|l|l|}
\hline $\begin{array}{l}\text { Motion } \\
\text { level }\end{array}$ & Slow & Medium & Fast & Total \\
\hline $\begin{array}{l}\text { Detected } \\
\text { Activity }\end{array}$ & $\begin{array}{l}76.6 \% \\
(2782)\end{array}$ & $\begin{array}{l}18.0 \% \\
(654)\end{array}$ & $\begin{array}{l}5.4 \% \\
(196)\end{array}$ & $\begin{array}{l}100 \% \\
(3632)\end{array}$ \\
\hline
\end{tabular}

detected activity within each hour based on the CFAR process. This gives a more intuitive picture about the activity level of the office. The rush hour in that day starts from 10AM until $3 \mathrm{PM}$ with a peak at 1PM and becomes less active after 5PM. There is a drop at 12PM which is believed due to the rest at lunchtime. The precise time of each activity can be extracted by zooming into the spectrogram for further analysis.

Afterward, according to the observations in Fig 6 , we estimate the corresponding motion level as the bin location of Doppler pulse which is defined as: slow motion is within $\pm 5 \mathrm{~Hz}$, medium motion is greater than $5 \mathrm{~Hz}$, and multiple Doppler pulses in a single measurement represent fast motion. Table I gives the detected activity for each motion level. As can be seen, the slow motion has the highest portion at $76.6 \%$, whereas fast motion only occupies $5.5 \%$. These motion levels give a preliminary image of the person acting in that day and could be useful to healthcare providers to evaluate the potential patient risks. For example, less overall medium-fast motions over a course of days or weeks could indicate a reduction in overall physical activity.

\section{CONCLUSIONS}

In this paper, we focus on the WiFi based sensing during its idle status where only sparse beacon signal are available for use by a sensor system. In sparse mode, a modified CAF has been used to maximize the usage of beacon signal. In enhanced mode, an external device has been used to simulate a client device that generates a high density of probe response signal. The experimental results indicate that both modes have significant improvements over the traditional processing as indicated in Fig 5. Especially, the enhanced mode can even have similar performance as that in $\mathrm{WiFi}$ transmission status (shown in Fig 6) which provides an attractive solution to improve the sparse WiFi signal. Lastly, long-term monitoring has been presented in Fig 7 with clear trace between the active and inactive period. It is envisioned that the proposed system has the potential in various healthcare applications and has the ability to adjust its Doppler quality depending on internet usage and healthcare requirement.

\section{ACKNOWLEDGMENTS}

This work was funded under the OPERA Project, the UK Engineering and Physical Sciences Research Council (EPSRC), Grant EP/R018677/1.

\section{REFERENCES}

[1] C. J. Caspersen, K. E. Powell, and G. M. Christenson, "Physical activity, exercise, and physical fitness: definitions and distinctions for healthrelated research." Public health reports, vol. 100, no. 2, p. 126, 1985.

[2] S. Savazzi, S. Sigg, M. Nicoli, V. Rampa, S. Kianoush, and U. Spagnolini, "Device-free radio vision for assisted living: Leveraging wireless channel quality information for human sensing," IEEE Signal Processing Magazine, vol. 33, no. 2, pp. 45-58, 2016.

[3] Z. Zhou, C. Wu, Z. Yang, and Y. Liu, "Sensorless sensing with wifi," Tsinghua Science and Technology, vol. 20, no. 1, pp. 1-6, 2015.

[4] W. Wang, A. X. Liu, and M. Shahzad, "Gait recognition using wifi signals," in Proceedings of the 2016 ACM International Joint Conference on Pervasive and Ubiquitous Computing. ACM, 2016, pp. 363-373.

[5] H. Wang, D. Zhang, Y. Wang, J. Ma, Y. Wang, and S. Li, "Rt-fall: A real-time and contactless fall detection system with commodity wifi devices," IEEE Transactions on Mobile Computing, vol. 16, no. 2, pp. 511-526, 2017.

[6] X. Li, S. Li, D. Zhang, J. Xiong, Y. Wang, and H. Mei, "Dynamicmusic: accurate device-free indoor localization," in Proceedings of the 2016 ACM International Joint Conference on Pervasive and Ubiquitous Computing. ACM, 2016, pp. 196-207.

[7] I. Milani, F. Colone, C. Bongioanni, and P. Lombardo, "Impact of beacon interval on the performance of wifi-based passive radar against human targets," in 2018 22nd International Microwave and Radar Conference (MIKON). IEEE, 2018, pp. 190-193.

[8] K. Chetty, G. E. Smith, and K. Woodbridge, "Through-the-wall sensing of personnel using passive bistatic wifi radar at standoff distances," IEEE Transactions on Geoscience and Remote Sensing, vol. 50, no. 4, pp. 1218-1226, 2012.

[9] $\mathrm{Ni}$ usrp $2921 . \quad$ [Online]. Available: http://sine.ni.com/nips/cds/view/p/lang/en/nid/212995

[10] R. v. Nee and R. Prasad, OFDM for wireless multimedia communications. Artech House, Inc., 2000.

[11] W. Li, B. Tan, and R. Piechocki, "Passive radar for opportunistic monitoring in e-health applications," IEEE journal of translational engineering in health and medicine, vol. 6, pp. 1-10, 2018.

[12] S. M. Markalous, S. Tenbohlen, and K. Feser, "Detection and location of partial discharges in power transformers using acoustic and electromagnetic signals," IEEE Transactions on Dielectrics and Electrical Insulation, vol. 15, no. 6, pp. 1576-1583, 2008.

[13] K. Kulpa, "The clean type algorithms for radar signal processing," in Microwaves, Radar and Remote Sensing Symposium, 2008. MRRS 2008, Sept 2008, pp. 152-157.

[14] I. C. S. L. S. Committee et al., "Ieee standard for information technology-telecommunications and information exchange between systems-local and metropolitan area networks-specific requirements part 11: Wireless lan medium access control (mac) and physical layer (phy) specifications," IEEE Std 802.11^, 2007.

[15] W. Li, B. Tan, and R. J. Piechocki, "Wifi-based passive sensing system for human presence and activity event classification," IET Wireless Sensor Systems, vol. 8, no. 6, pp. 276-283, 2018. 